\title{
ANALISI DELLA STRUTTURA SPAZIALE E PIANIFICAZIONE DEL PAESAGGIO AGRO-FORESTALE: PROSPETTIVE D'INTEGRAZIONE
}

\author{
(*) SISFOR, Laboratorio di Inventari Forestali e Sistemi Informativi Geografici, Dipartimento di Scienze dell'Ambiente \\ Forestale e delle sue Risorse, Università della Tuscia, Viterbo \\ (**) Laboratorio di Ecologia e Geomatica Forestale, Dipartimento di Scienze e Tecnologie per l'Ambiente e il Territorio, \\ Università degli Studi del Molise, Isernia
}

L'ecologia del paesaggio offre un ampio spettro di tecniche d'analisi applicabili a cartografie di uso e copertura del suolo per derivare informazioni quantitative su una varietà di aspetti della configurazione spaziale del mosaico paesistico; tra gli altri, misure relative alla estensione, forma, e livello di frammentazione spaziale delle superfici forestali (o di altre categorie di habitat naturali e seminaturali) sono ritenute utili a rispondere a requisiti informativi tipicamente formulati nel contesto di politiche di conservazione della biodiversità.

Analisi della struttura del paesaggio possono anche supportare l'individuazione di ambiti territoriali omogenei per valore paesaggistico da individuare nel piano paesaggistico regionale, rispetto ai quali pianificare obiettivi di qualità paesaggistica e prescrizioni per la tutela e l'uso del territorio (vd. D. Lgs. 42/2004, Codice Urbani).

Le metodologie di analisi e il monitoraggio della struttura spaziale del paesaggio agro-forestale sono ormai numerose e consolidate; tuttavia, solo di recente i risultati di questi studi hanno cominciato a supportare e orientare politiche di pianificazione del territorio per la formulazione di strategie di sviluppo territoriale differenziate in rapporto alle esigenze di conservazione degli habitat e della biodiversità e di salvaguardia del paesaggio calibrate in base alle specificità dei singoli territori. In particolare, il tema delle reti ecologiche, è divenuto oggetto specifico di pianificazione all'interno degli strumenti di area vasta.

In questa prospettiva il presente contributo intende delineare alcune riflessioni su come raccordare le conoscenze dell'ecologia del paesaggio alla formulazione di obiettivi di salvaguardia e valorizzazione del paesaggio, tenendo conto della configurazione attuale del sistema delle pianificazioni aventi competenza in materia di governo del paesaggio. Focalizzando l'attenzione su le problematiche di governo del paesaggio più tipiche del paesaggio agro-forestale, vengono inoltre prospettate alcune considerazioni sul possibile ruolo della gestione forestale come strumento d'attuazione delle previsioni inerenti la tutela e la riqualificazione del paesaggio espresse dagli strumenti di pianificazione di area vasta.

Parole chiave: ecologia del paesaggio, pianificazione territoriale, rete ecologica. Key words: landscape ecology, land planning, ecological network.

Mots clés: écologie du paysage, planification du territoire, réseau écologique.

\section{IL PAESAGGIO IN CHIAVE ECOLOGICA: LETTURA STRUTTURALE E DINAMICO FUNZIONALE DEL MOSAICO PAESISTICO}

L'ecologia del paesaggio è una disciplina scientifica relativamente recente, ma già ben articolata al suo interno in un corpus di principi teorici e strumenti di indagine che consentono di effettuare analisi e valutazioni sulla struttura e stato di funzionalità ecologica del paesaggio. Alla base di questo corpus di conoscenze sta un concetto di paesaggio che si svincola tanto da criteri estetico-percettivi, che dall'approccio storico secondo cui il paesaggio può essere considerato «un'elaborazione culturale di uno specifico ambiente naturale» (Sereni, 1983). L'ecologia del paesaggio assume il paesaggio come «un mosaico di ecosistemi e di usi del suolo che si ripete secondo una configurazione spaziale riconoscibile su un'area più o meno estesa» (Forman e Godron, 1986).

L'aspetto più innovativo introdotto da questa definizione sta nel riconoscere nel mosaico territoriale un vero e proprio livello organizzativo della gerarchia dei sistemi biologici, le cui componenti (comunemente indicate con il ter- mine di tessere) sono sistemi caratterizzati da differenti gradi di antropizzazione/naturalità e indicativamente riferibili al livello organizzativo dell'ecosistema.

Come in tutti i livelli di organizzazione biologica, il paesaggio è dotato di una struttura propria, che conferisce al paesaggio riconoscibilità. Infatti, la forma e la distribuzione spaziale delle tessere nel mosaico paesaggistico generalmente non è casuale, ma presenta caratteri di ripetitività che possono essere inquadrati secondo un modello o configurazione strutturale. Il modello più conosciuto e utilizzato è il cosiddetto modello dell'ecomosaico basato sul riconoscimento di tre tipologie di tessere: macchia, corridoio e matrice paesistica (Foto 1), a cui possono essere attribuite altrettante funzioni all'interno dell'ecomosaico (Tabella 1).

Esiste ormai un ampio spettro di tecniche d'analisi applicabili a cartografie di uso e copertura del suolo per esaminare e classificare la configurazione spaziale del mosaico paesistico. A esempio, a partire da immagini raster della copertura forestale (o di altre tipologie di habitat) è possibile attribuire mediante un algoritmo automatico di classificazione (http://forest.jrc.ec.europa.eu/biodiversity/ GUIDOS/) le tessere in esame a macchie di varia dimen- 
sione (aree nucleo-core areas e macchie) e strutture di collegamento (corridoio, ramificazioni) a differente valenza ecologica (Figura 1, Tabella 2). Questo tipo di analisi ha un evidente interesse applicativo nel contesto della pianificazione delle reti ecologiche.

Una rete ecologica, infatti, ha come obiettivo quello di garantire a una specie la piena funzionalità in ecomosaici ove il processo di trasformazione del territorio, la frammentazione e l'isolamento degli habitat naturali, nonché la diminuzione della loro estensione e qualità ambientale, potrebbe minacciarne la sopravvivenza. L'individuazione di un rete ecologica specie-specifica richiede tanto una lettura strutturale che dinamico-funzionale del mosaico territoriale, basato su conoscenze della biologia della specie (cf. Battisti, 2004). Infatti una stessa configurazione strutturale di ecomosaico può presentare differente funzionalità connettiva a seconda della specie considerata. In particolare, esistono differenze sostanziali tra la maggior parte degli invertebrati terrestri e dei vertebrati nella scala di utilizzo del paesaggio; tra le più rilevanti: $i$ ) una percezione diversa della dimensione degli habitat colonizzati, per i differenti ordini di grandezza nelle dimensioni corporee (cf. dimensione delle tessere); $i$ ) differenti livelli di specializzazione trofica e funzionale (cf. qualità dell'habitat); iii) differenti ordini di grandezza nel numero di specie per tipologia di ecosistema e nel numero di individui per popolazione locale; $i v)$ diverse capacità di risposta alle variazioni ambientali (cf. disturbi legati all'effetto di margine); v) differenti capacità di dispersione attiva e passiva.

Per questa ragione è più corretto parlare di reti ecologiche, composte da tessere specie-specifiche, individuate mediante modelli di habitat suitability (Corona et al., 2008).

Sulla base della conoscenza delle esigenze ecologiche di determinate specie scelte in quanto rappresentative del più vasto numero possibile di specie animali o vegetali (umbrella species) è possibile creare modelli matematici attraverso $\mathrm{i}$ quali valutare la potenziale funzionalità delle diverse tessere del paesaggio a svolgere la funzione di habitat per la specie analizzata. Tali approcci possono essere implementati su una logica booleana che permette di identificare se le tessere possano svolgere o meno la funzione di habitat o sulla base di più complessi approcci sfocati (fuzzy) che permettono di valutare la distanza ecologica tra l'attuale condizione di naturalità/disturbo della tessera e la sua teorica condizione di optimum ecologico per la specie selezionata.

L'applicazione più rilevante di questo approccio nel nostro Paese è la Rete Ecologica Nazionale (REN, Boitani et al., 2002).

Quando la rete ecologica diventa oggetto di pianificazione territoriale, non si può limitare l'attenzione su una o poche specie; gli orientamenti attuali in materia tendono a valutare la struttura e la funzionalità ecologica del mosaico territoriale nel suo insieme; l'obiettivo è rendere il territorio nel suo complesso idoneo alla presenza del maggior numero di specie e di habitat; ciò si realizza elaborando indirizzi per il governo del territorio e interventi utili a migliorare la connettività (o permeabilità biologica) dell'ecomosaico, ovvero la sua capacità di ospitare specie animali e vegetali e di assicurarne la mobilità e capacità di dispersione. Si parla in questo senso di reti ecologiche specie-aspecifiche o reti ecologiche territoriali (Bernetti e Chirici, 2005; Chirici, 2005). In questo senso, la presenza di naturalità diffusa nel territorio è considerata un presupposto necessario al buon funzionamento anche delle reti ecologiche specie-specifiche.

Da questa breve panoramica, appare chiaro che strumenti analitico-ricognitivi dell'ecologia del paesaggio, possono avere ricadute operative di grande interesse nel campo della pianificazione territoriale; ci si riferisce in particolare a quell'insieme di piani e programmi di area vasta in grado di influire sull'assetto del paesaggio, disciplinandone le trasformazioni e formulando indirizzi gestionali per la sua manutenzione. L'Italia si è peraltro recentemente dotata di uno strumento specifico per il governo del paesaggio, il piano paesaggistico regionale (D. Lgs. 42/2004, Codice Urbani), che diventerà un interlocutore fondamentale per tutti gli strumenti di pianificazione territoriale in grado di disciplinare le dinamiche evolutive del paesaggio (Piani Territoriali di Coordinamento Provinciale, PTCP) o implementare politiche di spesa in grado di influenzarne le dinamiche evolutive (es. Piani di Sviluppo Rurale).

In questa prospettiva, il presente contributo intende delineare alcune riflessioni su come raccordare le conoscenze dell'ecologia del paesaggio alla formulazione di obiettivi di salvaguardia e valorizzazione del paesaggio, tenendo conto della configurazione attuale del sistema delle pianificazioni aventi competenza in materia di governo del paesaggio.

\section{ECOLOGIA DEL PAESAGGIO E PIANIFICAZIONE DI AREA VASTA}

L'appartenenza a modelli strutturali di paesaggio differenti comporta problematiche gestionali diverse sotto il profilo del governo del paesaggio.

Il piano paesaggistico regionale è lo strumento chiamato a individuare sull'intero territorio regionale ambiti territoriali omogenei per valore paesaggistico rispetto ai quali definire obiettivi di qualità paesaggistica; questi si concretizzano in prescrizioni ed indirizzi tesi a attuare la tutela o la riqualificazione del paesaggio. Il piano opera in una dimensione prettamente regolamentare: esso fornisce un quadro organico di riferimento per articolare, strategie e discipline di maggior dettaglio per la tutela del paesaggio all'interno dei PTCP.

Di qui una prima considerazione. Gli strumenti d'analisi dell'ecologia del paesaggio risultano particolarmente funzionali all'identificazione di ambiti territoriali omogenei rispetto ai quali articolare le indicazioni e gli indirizzi di tutela, recupero e valorizzazione del paesaggio.

In particolare la metodologia della classificazione gerarchica del territorio (Blasi et al., 2000), permette l'individuazione di (un numero limitato di) ambiti territoriali considerabili omogenei da un punto di vista ecologico a seconda delle scale di rappresentazione (Figura 2). Al livello di maggior dettaglio sono individuate le Unità ambientali (scala 1:25000/1:5000) che identificano ambiti territoriali potenzialmente atti a ospitare un solo tipo di vegetazione seriale (serie di vegetazione); di fatto, per effetto dell'antropizzazione nel mosaico paesistico reale questi elementi di vegetazione possono non essere presenti o presentarsi in forma frammentata, all'interno di una matrice paesistica costituita da differenti tipi di coltivazione e di pattern insediativo e infrastrutturale.

Per quantificare in modo speditivo e secondo criteri og- 
gettivabili la qualità ambientale e lo stato di conservazione del paesaggio all'interno delle Unità ambientali, si può far riferimento invece all'indice di conservazione del paesaggio (Index of Landscape Conservation - ILC, Pizzolotto e Brandmayr, 1996). Si tratta di un indice sintetico ottenuto attribuendo un valore ordinale di stato di conservazione alle singole classi della legenda Corine Land Cover, tenendo conto di diversi livelli di alterazione del mosaico paesistico per effetto dell'antropizzazione (impermeabilizzazione del suolo, stato emerobiotico) e della vicinanza alla tappa matura delle aree naturali e seminaturali.

Seconda considerazione. L'articolazione del territorio in unità ambientali e la valutazione sintetica dello stato di conservazione delle singole unità, consente di articolare la disciplina normativa in indirizzi puntuali di tutela e di riqualificazione coerenti con l'identità naturalistica e storicoculturale dei luoghi.

Terza considerazione. L'ecologia del paesaggio appare la disciplina più adatta a coniugare la materia governo del paesaggio al tema della pianificazione delle reti ecologiche; la mappatura della rete ecologica a scala provinciale e la formulazione di indirizzi gestionali per il potenziamento della funzionalità della rete (vd. concetto di naturalità diffusa) sta emergendo come contenuto specifico dei PTCP; per la scala territoriale entro cui operano e la capacità di poter gestire temi ambientali differenti sul territorio provinciale $\mathrm{i}$ PTCP sono strumenti più abilitati a coordinare $i$ processi di trasformazione del territorio secondo strategie e azioni di sistema, rendendo possibile il confronto e il coordinamento fra strategie anche conflittuali operanti sul territorio provinciale: es. sviluppo delle reti di servizio e trasporti o del sistema insediativo e tutela dei paesaggi rurali.

Un esempio, in tal senso è offerto dal recente Piano Territoriale Provinciale Generale di Roma, che attraverso una lettura strutturale-territoriale del mosaico paesistico provinciale e sulla base di conoscenze puntuali sulla distribuzione della flora e della fauna (specie di importanza conservazionistica e biogeografica) ha cartografato la Rete Ecologica Provinciale (REP), con l'obiettivo di tutelare la funzionalità e l'efficienza del mosaico territoriale in termini ecologici, nei suoi aspetti strutturali (flora, vegetazione, fauna e paesaggio) e funzionali (connessioni, connettività e permeabilità).

\section{RAPPORTI TRA IL GOVERNO DEL PAESAGGIO E LA GESTIONE} FORESTALE

Da quanto precedentemente delineato è possibile tracciare alcune considerazioni sul possibile ruolo della gestione forestale come strumento d'attuazione delle previsioni di tutela e la riqualificazione del paesaggio espresse dagli strumenti di pianificazione di area vasta. Sotto il profilo degli obiettivi, gli interventi di gestione forestale possono essere riferiti a tre scenari operativi distinti:

- conservazione, intesa come applicazione di forme di gestione attiva dei boschi (selvicoltura sistemica, mantenimento/recupero delle attività selvicolturali tradizionali) utili a mantenere o migliorare lo stato di efficienza bioecologica delle tessere forestali che, nella gran parte dei casi, rappresentano l'ossatura principale delle reti ecologiche individuate a scala territoriale; di particolare rilevanza per il mantenimento di particolari valori paesaggistici, storici e culturali connessi alla presenza di tessere forestali può essere anche la scelta di continuare (o recuperare) pratiche selvicolturali tradizionali che hanno prodotto tessere forestali di riconosciuto valore (es. abetine storiche di Vallombrosa, castagneti da frutto, sugherete, pinete costiere);

- riqualificazione, intesa come insieme di interventi volti al recupero di condizioni di maggior naturalità nelle tessere forestali semplificate nella struttura e nella composizione (rinaturalizzazione) e alla riduzione della frammentazione degli habitat naturali: es. favorire l'evoluzione delle formazioni preforestali coerenti con la vegetazione forestale, prevedere l'impianto di nuovi boschi o arbusteti, in aree critiche in termini di connettività e funzionalità della rete ecologica territoriale;

- valorizzazione del paesaggio agricolo, azioni volte alla reintroduzione di isole di naturalità diffusa in territori dominati da matrice agricola: misure per migliorare la connettività della matrice anche con interventi lineari (siepi, frangivento, fasce arborate ripariali utili anche in funzione di fitodepurazione) e/o arealmente poco estesi (nuclei di boschi e arbusteti con funzione di stepping stones) sulle superfici agricole non più utilizzate e nelle piccole parti del sistema agricolo (tare). In questi contesti, l'introduzione di nuovi boschi può presentare importanti ricadute anche in termini di mitigazione dei fenomeni di diffusione urbana nella matrice agricola; isole di naturalità, opportunamente posizionate nell'interfaccia matrice agricola-urbano costituiscono un presidio contro il pericolo di occlusività da nuove costruzioni, riducendo il grado di trasformabilità della matrice agricola ed esaltandone la percettibilità.

\section{RIFLESSIONI CONCLUSIVE}

L'ecologia del paesaggio rappresenta una disciplina ormai matura per assumere un ruolo non solo di tipo descrittivo e conoscitivo, ma anche pianificatorio nel contesto degli strumenti di governo del territorio.

L'inserimento delle tematiche di tutela del paesaggio e delle reti ecologiche nella pianificazione di area vasta investe la gestione forestale di nuovi obiettivi e responsabilità, che non si limitano a un concetto di manutenzione o conservazione attiva del paesaggio forestale. Soprattutto in ecomosaici dominati da una matrice agricola l'introduzione di isole di naturalità diffusa, nella forma di boschi e arbusteti, in aree critiche dal punto di vista della connettività, così come individuate da analisi di rete ecologica, diventa strumento dalle significative potenzialità per la riqualificazione ecologica del paesaggio. Sotto il profilo degli effetti gli interventi di gestione forestale sopra indicati possono assolvere infatti a funzioni sinergiche e trasversali in quanto consentono:

- l'avvio di un processo di recupero dell'identità naturalistica del territorio, attraverso l'impianto nella matrice agricola, di tessere di vegetazione forestale di piccola estensione; - di potenziare con nuovi elementi strutturali la funzionalità della rete ecologica territoriale questo tipo d'interventi si configurano come stepping stones e possono inoltre andare a costituire nel tempo eventuali fonti di seme per processi di colonizzazione naturale, che si potrebbero attivare spontaneamente a carico della matrice agricola, in caso di abbandono della coltivazione;

- di mitigare i processi di diffusione urbana nella matrice agricola, si tratta di un argomento evidentemente comples- 
so - che può apparire provocatorio alla pianificazione urbanistica tradizionale, rivolta a trasformare tutto lo spazio aperto in area edificabile - ma che rappresenta invece un tema di grande attualità nei piani regolatori comunali di nuova generazione; essi manifestano una crescente sensibilità alle tematiche di gestione e salvaguardia del verde urbano nella trasformazione della città, tanto da utilizzare cartografie della rete ecologica come elaborati prescrittivi e invarianti della pianificazione comunale.

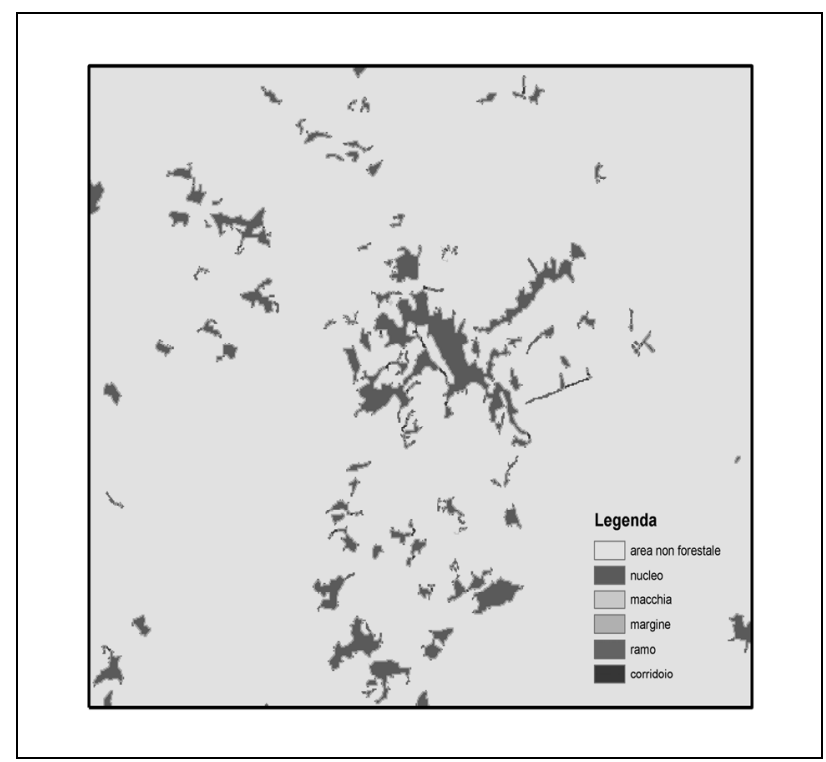

Figura 1. Esempio di classificazione del pattern strutturale della copertura forestale di un'area della Sicilia centrale; per dettagli sulle classi vd. Tabella 2 (elaborazione tramite software GUIDOS http://forest.jrc. ec.europa.eu/biodiversity/GUIDOS/ su dati Corine Land Cover 2000).
In questa prospettiva è opportuno stabilire e rafforzare l'integrazione verticale e orizzontale tra gli strumenti di pianificazione territoriale di area vasta (Piano Paesistico Regionale e Piani Territoriali di Coordinamento Provinciale), gli strumenti di pianificazione forestale di livello regionale e sub-regionale (Piani Forestali Regionali, Piani Forestali di Indirizzo Territoriale) e le politiche di spesa dei Programmi per lo Sviluppo Rurale.

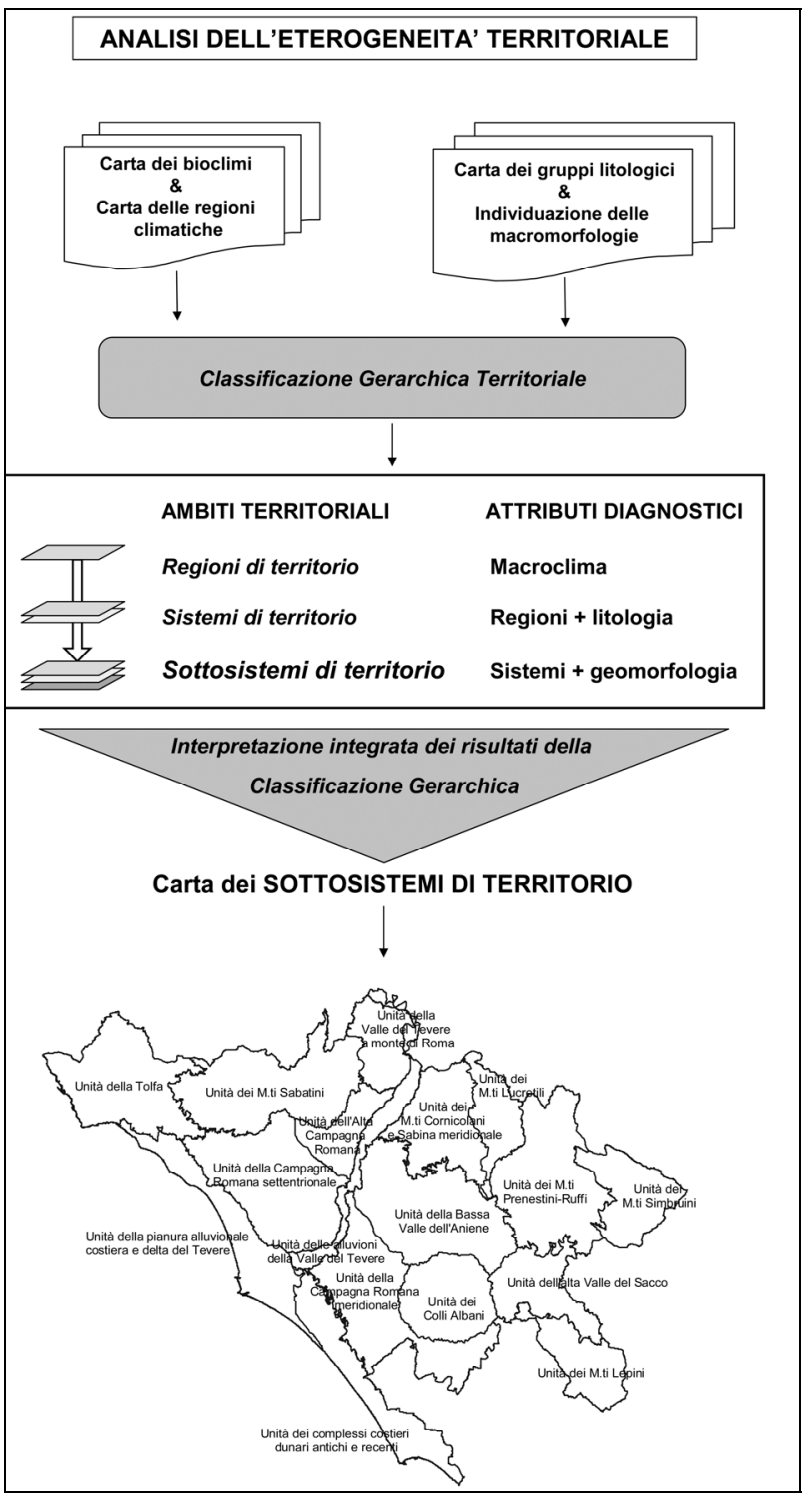

Figura 2. Piano Territoriale Generale Provinciale (PTGP) della Provincia di Roma: esempio di applicazione della classificazione gerarchica del territorio all'individuazione di Unità territoriali ambientali, utilizzate come unità di base per il governo del territorio (Fonte: http://ptpg.provincia.roma.it:8080/). 


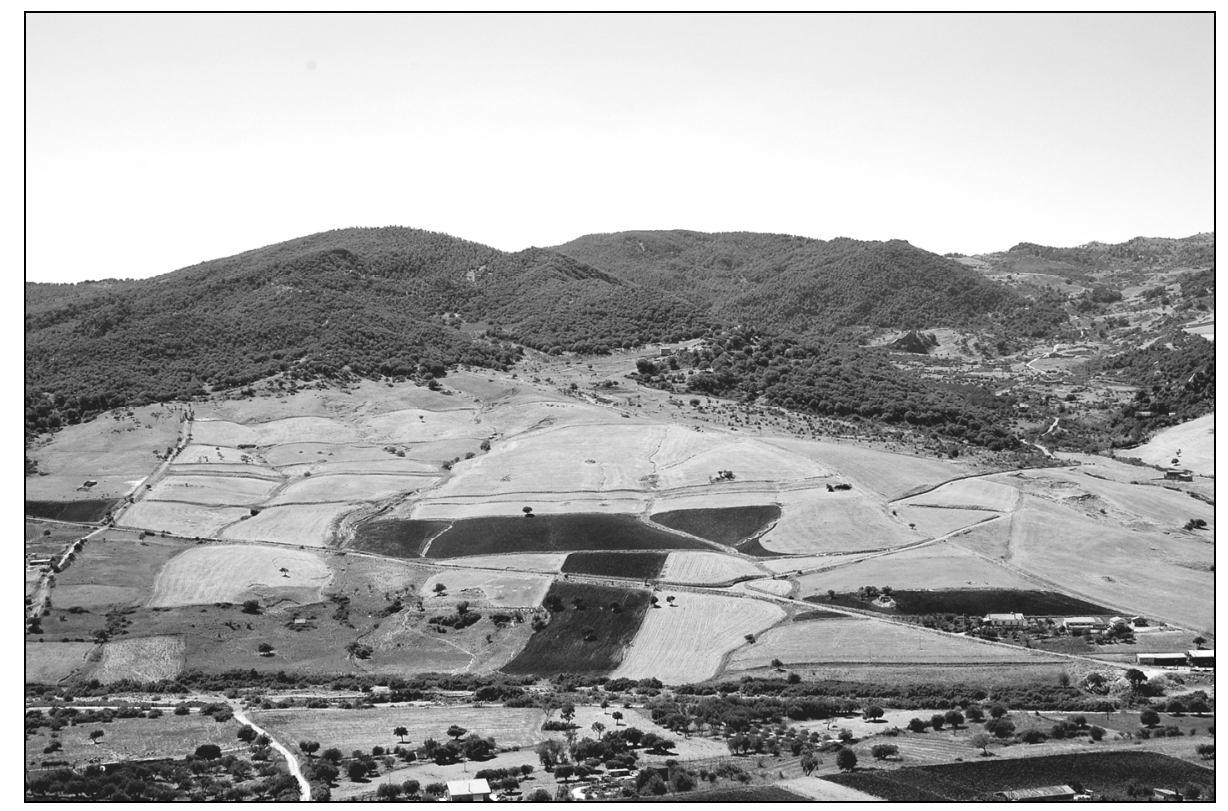

Foto 1. Sicilia: tipologie diverse di eco mosaici nei territori agro-forestali: la fascia alta del rilievo è dominata da una matrice paesistica di tipo forestale, punteggiata da piccole macchie (aree aperte, coltivi); nella fascia basale e nel fondovalle, la matrice diventa agricola (prevalentemente seminativi), attraversata da corridoi nella forma di fasce di vegetazione e viabilità (foto: T. La Mantia).

\begin{tabular}{|c|c|c|}
\hline $\begin{array}{c}\text { Componente } \\
\text { dell'ecomosaico }\end{array}$ & Caratteristiche strutturali & Funzioni principali \\
\hline Macchia & $\begin{array}{c}\text { Tessera senza una dimensione spaziale prevalente che } \\
\text { differisce dal mosaico paesistico circostante }\end{array}$ & $\begin{array}{c}\text { Conservazione della biodiversità: habitat per specie vegetali } \\
\text { e animali, rifugio, sorgente/sink (metapopolazioni) }\end{array}$ \\
\hline Corridoio & $\begin{array}{c}\text { Tessera con sviluppo lineare più o meno stretta, ele- } \\
\text { mento di connessione tra macchie di tipo analogo (o } \\
\text { viceversa barriera), che differisce in modo netto dalla } \\
\text { matrice che lo circonda su entrambi i lati }\end{array}$ & $\begin{array}{c}\text { Nel caso di fasce di vegetazione: } \\
\text { Habitat/Condotto/rifugio per specie vegetali e animali } \\
\text { Filtro (es. fitodepurazione) } \\
\text { Nel caso di strade/infrastrutture, possibile barriera }\end{array}$ \\
\hline Matrice & $\begin{array}{c}\text { Elemento paesistico più estensivo e/o più interconnesso } \\
\text { del mosaico paesistico }\end{array}$ & $\begin{array}{c}\text { Controllo della dinamica del mosaico paesistico, ovvero dei } \\
\text { flussi di materia e energia (es. sequestro del carbonio, con- } \\
\text { servazione del suolo e delle acque) e informazione (es. pro- } \\
\text { cessi genetici) }\end{array}$ \\
\hline
\end{tabular}

Tabella 1. Caratteristiche strutturali e funzionali delle componenti principali dell'ecomosaico.

\begin{tabular}{|c|c|c|}
\hline Classe strutturale sensu GUIDOS & Caratteristiche strutturali & Elemento di rete ecologica \\
\hline Aree nucleo o centrali (core) & $\begin{array}{l}\text { Bosco (o altro habitat naturale e seminaturale) suffi- } \\
\text { cientemente esteso da poter essere suddiviso in una } \\
\text { area di margine, di transizione con la matrice ester- } \\
\text { na, e in un settore interno, detto nucleo, ove gli effet- } \\
\text { ti dovuti alla matrice esterna non intervengono o } \\
\text { agiscono in misura minima }\end{array}$ & $\begin{array}{c}\text { Aree centrali (core areas) superfici con presen- } \\
\text { za di biotopi, habitat naturali e seminaturali con } \\
\text { alto contenuto di naturalità, un elevato numero } \\
\text { di specie e/o una popolazione di particolare in- } \\
\text { teresse biogeografico e conservazioni stico; } \\
\text { coincidenti con aree già sottoposte o da sotto- } \\
\text { porre a tutela }\end{array}$ \\
\hline Macchia (patch) & $\begin{array}{l}\text { Boschi (o altri habitat naturali e seminaturali) isolati } \\
\text { di dimensione troppo piccola per contenere aree nu- } \\
\text { cleo }\end{array}$ & $\begin{array}{l}\text { Nuclei di connessione (stepping stones) punti di } \\
\text { connessione in grado di sostituire i corridoi }\end{array}$ \\
\hline Margine (edge) & $\begin{array}{l}\text { Superficie perimetrale del bosco (o altro habitat na- } \\
\text { turale e seminaturale), di transizione tra il bosco e le } \\
\text { superfici contermini }\end{array}$ & $\begin{array}{c}\text { Zone cuscinetto (buffer zones) zone e fasce a- } \\
\text { diacenti alle aree centrali che costituiscono il } \\
\text { collegamento tra attività antropiche e dinamiche } \\
\text { naturali }\end{array}$ \\
\hline Ramo (branch) & Ramificazione lineare innestata su aree nucleo & - \\
\hline Corridoio (corridor) & $\begin{array}{l}\text { Bosco (o altro habitat naturale e seminaturale) di } \\
\text { collegamento tra aree nucleo }\end{array}$ & $\begin{array}{l}\text { Corridoi di connessione (green ways/blue ways) } \\
\text { ambiti finalizzati alla dispersione delle specie }\end{array}$ \\
\hline
\end{tabular}

Tabella 2. Classi strutturali derivate dal software GUIDOS e possibili relazioni con componenti delle rete ecologica territoriale. 


\section{SUMMARY}

\section{ANALYSIS OF LANDSCAPE STRUCTURE AND RURAL PLANNING: PERSPECTIVES OF INTEGRATION}

Landscape ecology has developed a broad range of techniques to extract from land use and land cover maps quantitative information on landscape spatial configuration; amongst others, the size, shape and level of fragmentation of forest areas (or of other types of natural and semi-natural habitats) are regarded useful variables to be assessed and monitored in the framework of biodiversity conservation policies.

Landscape structural analyses are also valuable for delineating and mapping landscape units relevant for planning actions aimed at landscape protection and restoration, activities recently regulated in Italy by the national law D. Lgs. 42/2004.

Today, there is a plenty of well-established methods for analyzing and monitoring landscape spatial structure. Yet, only recently have these kind of studies become a decisionsupport tool for land planning policies and instruments, e.g. for addressing issues of biodiversity conservation through ecological network planning.

In this perspective, the paper outlines some examples on how instruments of land and landscape planning currently in force in Italy, would benefit from the support of landscape ecology tools to address goals of landscape and biodiversity protection. The attention is focused on planning issues typical of rural landscapes. Thoughts are given on the potential role of forest management in supporting the practical implementation of landscape protection strategies, including the enhancement of local ecological networks.

\section{RÉSUMÉ}

\section{ANALYSES DE LA CONFIGURATION DU PAYSAGE ET PLANIFICATION DU TERRITOIRE RURALE: PERSPECTIVES D' INTEGRATION}

Les techniques d'écologie du paysage fournissent une ample gamme d'instruments opérationnels, pour l'évaluation potentielle de la fonctionnalité écologique, des environnements naturels et semi-naturels, ceci sur la base de leur configuration dans l'espace. Ces techniques se basent sur l'élaboration de cartographies des habitats forestiers ou, plus en général, des milieux naturels ou semi-naturels. De telles techniques permettent de dériver des informations thématiques relatives à un vaste nombre d'indicateurs, comme l'extension, la forme, la fragmentation des environnements naturels et semi-naturels.

$\mathrm{Au}$ niveau Européen et global différents processus internationaux reconnaissent l'importance des informations qui découlent des analyses d'écologie du paysage pour la réduction et la mitigation des effets de la fragmentation et de la dégradation des habitats naturels et semi-naturels.

Les nombreuses initiatives destinées au monitorage de la configuration de l'espace des systèmes forestiers, ont habituellement des finalité cognitives, mais elles permettent rarement d'orienter activement les choix de la planification $\mathrm{du}$ territoire. L'écologie du paysage et planification du territoire opèrent à des degrés différents avec un niveau marginal d'intégration.

Cette contribution illustre certains résultats récemment obtenus lors d'une série analyses d'écologie du paysage in Italie. À partir de ces résultats sont prospectées solutions opérationnelles de planification du territoire et du paysage rural, qui sont orientées vers le renforcement de réseaux écologiques, avec le support de la gestion forestière.

\section{BIBLIOGRAFIA}

Battisti C., 2004. Frammentazione ambientale, connettività, reti ecologiche. Un contributo teorico e metodologico con particolare riferimento alla fauna selvatica. Provincia di Roma, Assessorato alle politiche ambientali, agricoltura e protezione civile, Roma.

Blasi C., Capotorti G., Smiraglia D., Frondoni F., Ercole S., 2005. Percezione del paesaggio: identità e stato di conservazione dei luoghi. In: Blasi C., Paolella A. (a cura di) "Identificazione e cambiamenti nel paesaggio contemporaneo", Documento IAED, N. 24, Edizioni Papageno, Palermo, pp. 13-22.

Blasi C., Carranza M.L., Frondoni R., Rosati L., 2000. Ecosystem classification and mapping: a proposal for Italian landscapes. Applied Vegetation Science, 2: 233-242.

Boitani L., Corsi F., Falcucci A., Maiorano L., Marzetti I., Masi M., Montemaggiori A., Ottaviani D., Reggiani G., Rondinini C., 2002. Rete Ecologica Nazionale. Un approccio alla conservazione dei vertebrati italiani. Università di Roma "La Sapienza", Dipartimento di Biologia Animale e dell'Uomo; Ministero dell'Ambiente, Direzione per la Conservazione della Natura; Istituto di Ecologia Applicata.

Chirici G., 2005. Analisi della rete ecologica territoriale nazionale: prodromi di un approccio sfocato su base GIS. In "Foreste Ricerca Cultura - scritti in onore di Orazio Ciancio", (Corona P., Iovino F., Marchetti M., Menguzzato G., Nocentini S., Portoghesi L. a cura di). Accademia Italiana di Scienze Forestali, Firenze, pp. 99-122.

Bernetti I., Chirici G., 2005. La rete ecologica del circondario Empolese-Val d'Elsa: analisi e strumenti di gestione. Atti della IX conferenza ASITA, 15-18 novembre 2005, Catania: 333-338.

Corona, P., Salvati, R., Barbati, A., Chirici, G., 2008. Land suitability for short rotation coppices assessed through fuzzy membership functions. In "Patterns and processes in forest landscapes. Multiple use and sustainable management" (R. Lafortezza, J. Chen, G. Sanesi \& T.R. Crow, Eds.): 168-191. Springer.

Forman R.T.T., Godron M., 1986. Landscape ecology. New York, Wiley.

Pizzolotto R., Brandmayr P., 1996. An index to evaluate landscape conservation state based on land-use pattern analysis and geographic information techniques. Coenoses, 11: 37-44.

Sereni E., 1983. Il paesaggio. La Nuova Italia, Roma. 